\title{
COMPUTER GAMING, BIOTECHNOLOGY AND ARCHITECTURE: EMBEDDING THE INTERSECTION WITHIN AN ARCHITECTURAL CURRICULUM
}

\author{
RUSSELL LOWE \\ Faculty of the Built Environment \\ University of New South Wales, Sydney \\ NSW 2052, Australia \\ russellL@fbe.unsw.edu.au
}

\begin{abstract}
Today, leading computer games provide real time environments including spaces, objects and characters that range (by manipulating an enormous array of parameters and being subject to simulations of real world physics) from the super realistic to the super delirious. Biotechnology, although apparently unrelated, also requires the manipulation of information in space and time and promises to affect environments in a range of ways that is at least as extreme. The opportunities suggested by an intersection between Architecture, Computer Gaming and Biotechnology were instrumental in the creation of courses and topics for students in first year right through to students studying toward a Masters degree.

This paper reflects on and critically reviews the implementation, strategies and outcomes of embedding the intersection between Computer Gaming and Biotechnology within an Architectural curriculum. It draws from the experience of over 500 students, two Universities and major technological shifts. It develops the notion of the experiment in design.

In contrast with the introduction of computer gaming technology into a core first year course, that had the underlying aim of including these technologies as a part of a general design curriculum, the introduction of issues connecting architecture with biotechnology (through computer gaming technology) reflects the specific research agenda of the author and is not intended for general application across an architectural curriculum. For more general application it could be seen as a strategy to promote cross disciplinary collaboration through the concept of the "boundary object'.
\end{abstract}

Keywords: Architecture, Computer gaming, Biotechnology, Design Experiment, Boundary Object. 


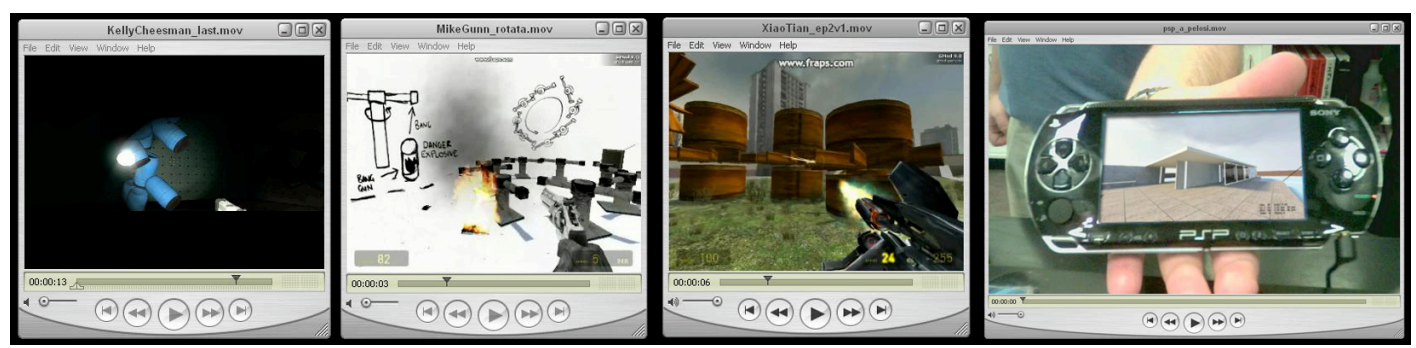

Figure 1: Examples of student work - Kelly Cheesman, Michael Gunn, Xiao Tian, Antony Pelosi.

\section{Introduction}

A recent article in 3D World drew attention to a trend that computer game "modders" could see coming from some time ago.

"This year's event continued the evolution that began in 2005: architectural visualization now makes up about half of the program, while the video game industry has been somewhat sidelined. And yet, in an interesting twist, video game technologies were to the fore in nearly all of the event's conferences and special presentations."

They go on to say that "the various presentations made it quite obvious that simulations of light, fluids and behaviour have reached such a high level of realism and effectiveness that they are on the verge of supplanting all other traditional approaches" (Imagina 20).

When the authors suggests that the realism and effectiveness of these simulations is on the verge of supplanting traditional methods they are writing with regard to professionals within the animation/visualization industry. In terms of the capacity of the general undergraduate student to create these simulations, computer gaming technologies will allow them to far exceed their capabilities than if they were restricted to traditional CAD approaches. In 2003 the idea of computer gaming technologies supplanting traditional approaches within an architectural curriculum suggested an opportunity to rethink the way those traditional digital technologies contributed to the design process. This paper reflects on the implementation and development of that opportunity from 2003 to the present.

\section{DESN104: Introduction to Computers for Designers: Developed for Victoria University of Wellington, New Zealand. 2003-2006}

This first year multidisciplinary course formed a part of the core curriculum for students intending to enrol in further studies in Architecture, Interior Architecture, Landscape Architecture, Industrial Design and more recently Digital Media Design. A key requirement for the course was for students to develop strategies for choosing and learning software that would become instrumental in their exploration and experimentation in design. The inclusion of computer gaming technology within the curriculum facilitated this in two ways. It provided an additional category of digital modelling, to add to the traditional two of solid and surface modelling, thereby increasing the depth and richness in terms of comparisons and contrasts that could be made. See Figure 2 below. Secondly, the priority of the software is towards a real time interactive experience of design that shifts dramatically away from the preconceived outcomes that one associates with CAAD software and traditional key frame animation. This priority is absolutely necessary for scientific experimentation.

The inclusion of computer gaming technology within an architecture course is not unique, in fact Lehtinen (2002) claimed that "game engines have been tested in this purpose for at least a decade". He went on to say that the "only limiting factor was ease of use" which resulted in only a "select few" becoming acquainted with the technology (within an architectural visualisation course). Over three years he had a total of 7 students. While these numbers seem low they are actually quite typical. In 1999 Achten held a studio in Eindhoven, Germany, using 6 PC's and in 2002 Moloney held a studio with 26 students. In addition to their small numbers often the students in question were in the final stages of their degrees. In direct contrast to this the enrolments in DESN104 usually fluctuated between 90-120 students with almost all of them being first year students with no prior knowledge of $3 \mathrm{~d}$ modelling software. 
There were three reasons we were able to over come the obstacles that faced the other authors and introduce computer gaming technology so early and with such high numbers of students.

1. In 2002 Lehtinen said that "all game creation tools currently lack any easy way to import from any common CAD-software". 2003 saw a shift in approach to custom content from the game makers. The Unreal engine 2 with the Ued3, first released with UT2003, was the first major game engine/editor combination that would rely on outsourced models and textures. The developers at Epic Games see their Unreal Editor as a "content creation tool filling the void between 3D Studio Max and Maya, and shippable game content" (Unreal Technology).

2. As recently as 2004 O'Coill and Doughty found that models built using a combination of AutoCAD and 3DSMax with only 400 polygons would have over 100 errors. Repeated failures like this resulted in their $\mathrm{PhD}$ students giving up on working with a computer game engine altogether. The inclusion of a solid modelling software in the suite of software DESN104 students used totally eliminated errors due to open meshes and coincident surfaces (the cause of O'Coill and Doughty's problem). With the ultimate realisation of their design work being a computer gaming environment DESN104 students were instructed to use the surface modeller for modifying, texturing and UVW mapping geometry only, not creating it.

3. Ironically the final reason why we were able to introduce computer gaming technology so early and with such high numbers of students is that the students were at an early stage in their design education and that there were a large number of them. Many authors have found that significant prior knowledge of CAAD software can be an impediment to learning computer game editing software (O'Coill and Doughty, Hoon and Kehoe). In addition to this Zobel has noted that the facilities of $3 \mathrm{~d}$ visualisation and virtual reality "facilitate an increased understanding for those people who are unused to the traditional design tools" (1995). The large size of the class made it possible to take advantage of some of the opportunities suggested by the culture surrounding computer gaming technology outlined in section 4 below.

Figure 2 (below) shows a list of parameters that define the qualities that facilitate real time architectural experimentation using digital media. This table should not be understood as a description of three particular instruments but rather as a matrix of elements that might be combined to create a Synthetic Instrument (Stratton). Each of the generic instruments on the top row may be replaced by one of the many alternatives that exist in its category.

\begin{tabular}{|l|l|l|l|}
\hline instrument & SolidWorks & 3DSMax & UnrealEd3 \\
\hline parameter & solid modelling & surface modelling & spatial modelling \\
\hline Sculptural priority & simple & challenging & dispersed \\
\hline Tutorials & very small & large & massive \\
\hline Online community & industrial design & architectural & game design \\
\hline Industry & physicality & aesthetically & geometry \\
\hline Materiality & parametric & key framed & real time \\
\hline Interaction & none & via plug-ins & by design \\
\hline Modding & none & acting on geometry & environmental \\
\hline Sound & very expensive & expensive & free \\
\hline Cost & medium & high & low \\
\hline Complexity of Use &
\end{tabular}

Figure 2: Matrix of software elements that might combine to create a Synthetic Instrument 


\section{The 'experiment' in DESN104; Introducing Scientific Method}

Following Matta-Clark, design is characterized as an imaginative disruption of convention. In each experiment students were expected to find and exploit possible causes of the disruption of convention by first developing a hypothesis that would be based on the observation of a designed object, space, terrain or relationship chosen from a limited pool. Each pool comprised a small group of artists, designers and companies who were well known for the disruptive effect their work has had with respect to the conventions of their creative communities. Artists, designers and companies such as Zaha Hadid, Peter Eisenman, Issy Miaki, Psyop, Patricia Piccinini, Michele Gondry and the Chapman Brothers are a representative sample of those that have supplied projects that have acted as hypothesis generating precedents within these experiments.

The best definition describing the scientific method in this first stage is provided by the Frascati Manual and elaborated under the category "Experimental development". Experiment development is defined by "systematic work, drawing on existing knowledge gained from research or practical experience, that is directed to ... new processes, systems and services; or to improving substantially those already produced..." (Frascati 7)

The first experiment in DESN104: Introduction to Computers for Designers, (Lowe) was conducted over a three week period that included six three-hour tutorial sessions. The experiment required students to select one project from the portfolio of Ron Arad, Herzog \& de Meuron, Michael Heiser, or Patricia Piccinini and generate a hypothesis from it that would challenge conventional understandings of normalcy and mutantcy. Using the Ued3 world builder they each created two rooms, a normal room and a mutant room, which were to be connected by a corridor. Along with a website that provided an executive summary of the environment they created each student submitted a UT2004 map that would provide the key evidence demonstrating specific qualities within their experimentation. The primary assessment of the work was carried out within the real time environment itself where tutors were looking to experience the imaginative disruption of convention the students had created.

Following a tutorial that shipped with a special edition of the UT2004 game DVD the student's were able to engage with the Ued3 world builder and begin experiencing the spaces they had designed within 15 minutes of the beginning their first class. Frequent 'play-testing', where the students 'compile' and enter the real time 3d version of their environment generated in Ued3, was emphasized as a strategy for testing and systematizing their work. The required sophistication of the students elaboration of conceptual terms (demanded by the transitional condition of the corridor) and the collateral learning taking place (creating custom textures for their environment in Adobe PhotoShop, capturing screen shots in Fraps for the website they designed in Macromedia DreamWeaver) set up an incredibly challenging experiment that was for many students their introduction to design computing. Even though many students found the learning curve very steep there seemed to be a profound sense of accomplishment as they experienced their architecture coming to life in 3 dimensions in real time. They were, literally as well as metaphorically, immersed in their work. This contrasts with the arms length engagement we've experienced when introducing traditional CAAD software. In June 2006 an independently conducted and collated "End-of-Course Evaluation by Students" found that $81 \%$ of students either agreed or strongly agreed that their "interest in the subject has been stimulated by the content and presentation of this course".

The second experiment, called "Making and Modifying", had students create a first object (Treadwell 1996) in SolidWorks, export it to 3DStudio Max and use a limited selection of modifiers to generate 81 additional versions. In addition to its conceptual underpinnings in terms of Originals/Copies/Multiples/Series and reflecting on the work of Rosalind E. Kraus this experiment set students up with the required skills and techniques for creating the outsourced $3 \mathrm{~d}$ models that they would use to fully reengage with the Ued3 world builder in the final experiment.

The final experiment, called Navigation, utilized these custom models as vehicles (both literally and figuratively) to have students experiment relativisticly; that is, to understand that perspective and point of view play a significant role in experimental observation. In their environment the students had to enable navigation below, on and above a terrain. Please see the link below for examples of student work (Lowe).

Examples of student work from experiment 3 "Navigation":

1. Oren Oaariki used an investigation of graffiti (inspired by Psyop) to subvert the conventional understanding of an arrow as a symbol for accurate navigation. His 
pathways became three dimensional scripts (each writing the word "space") that oscillated between floor, wall, and ceiling surfaces and always terminating with an arrow. While looking anything but, they were surprisingly navigable and demonstrated incredible control in terms of guidance through an open architectural network (a mobius strip is closed and simplistic in comparison). Designing such complex forms that rely on a finely balanced manipulation of the occupant's perception (itself changing over time) would rely on so much assumption and guesswork as to be almost irrelevant without frequent and immersive testing against experience.

2. Rurehe Taylor's intention was to recreate a historical event "Urapatu" (a NZ Maori word that translates to "scorched earth policy") by creating an environment of "fear and pain". His Architecture is created by fragments coming together after being triggered by occupants as they navigate the scorched landscape. His use of atmospheric effects including fog, fire and lightning created a parallel temporality in visual acuity. It seemed initially that a strong narrative might be at odds with a real time interactive environment (and better be expressed in a traditional animation). Rurehe's strategy of inflicting pain and death (in one's avatar) if one strayed from the path, and ultimately death at its conclusion left enough room for occupants to feel engaged but swept along by events that attained an undeniable certainty.

3. Hye Bin Sung created an environment inspired by a chest of drawers by Droog design (Tejo Remy, 1991). She interrogated the very notion of environment by reproducing it as a series of spaces that deal with environmental factors as independent elements. One space reversed the effects of gravity, another was filled entirely with water, a third used "emitters" to fill a space with multiple objects that moved without physical presence or resistance (their collision was disabled). Audio effects occupying specific zones throughout her 'environment' supported and extended the above. Frequent "playtesting" comparing multiple versions of the environment allowed Hye Bin to develop a sophisticated understanding of the subtleties between the experience of falling (in this case upwards) and floating through space.

\section{Learning from the Culture of the Computer Gaming Community}

In bullet pointing the positive characteristics of their new Unreal Engine Epic games highlights the importance of the "mod community".

"Every Unreal Engine license includes the right to redistribute UnrealEd publicly, enabling teams to release the content creation tools along with their game to the mod community. Mod support has been a major factor behind the success of many prominent PC games today, and we anticipate that support for PC-based mod development may be a significant factor in future console games as well" (Unreal Technology).

Modding a PC game can involve anything from changing the look of a character to creating a whole new environment to changing fundamental relationships between every active element within an environment (making gravity work upwards for example). They are produced by a largely volunteer community that is characterized by an open flow of information, free distribution of custom content and collaboration. The community grows and sustains itself through websites and forums dedicated to providing online training and answers for technical questions (3dbuzz.com is a good example). To a much, much, lesser degree they facilitate design critique.

Following this paradigm and paralleling the introduction of computer gaming technology the method of instruction shifted from 'demonstration' to 'collaboration' via small clusters we called research groups. Previously the course had employed the demonstration model where the course instructor, or tutor, would demonstrate a sequence of operations while students would take notes and then attempt to replicate the result themselves. Due to the length of many demonstrations it was inevitable that the student's recollection would be incomplete. The result was that after each demonstration the tutors would move from student to student filling in the gaps in their note taking and, more often than not, answer the same question many times over. With the collaborative research group model students were placed into research groups of approximately six students at the beginning of the course. The students in each research group were told that they were to rely on each other to gather the technical skills to complete each experiment. The tutors were instructed not to answer any technical question directly but were to facilitate the process whereby the students learned how to find the answer for themselves. Following the modding community often this involved collaboration, discovering websites, posting on online forums and learning to read and critique the tutorials available on them. This 
helped students directly regarding the computer gaming software they were using and indirectly in terms of mining the effective and not so effective help functions of more conventional CAD software (Solidworks and 3dsMax respectively).

Both tutors and students found this approach difficult at first, often it would have been much easier to indicate a certain button to press or certain step that the student was missing. But quite quickly it became apparent that the research group provided a vehicle where every student had something distinct to contribute, even if it were simply the clarity with which they formulated their questions. With this approach the tutors found they could become higher level facilitators focusing on pedagogy rather than micro managing skill acquisition. It followed that students were able to spend more time designing and the tutors more time advising on design development.

An important part of the design development process involved in-game critiques of the students work. At various times students were required to swap environments with each other and complete the same marking schedule that the tutors would later use to grade their work. On other occasions the whole session (30-45 students) would explore one map simultaneously. Where one student navigating a map (without prior coaching) provided interesting anecdotal evidence as to the clarity, or otherwise, of its experiences and "pathways" the presence of a large group within the environment established clear trends that we found were replicated in other tutorial sessions. For many it was a surprise to learn how people actually navigated their spaces. We found that students productively assimilated feedback from these sessions in their subsequent design work.

\section{ARCH1501 Investigation Workshop, Experimenting within the Intersection between Architecture and Biotechnology. Developed for UNSW FBE 2007.}

Background: ARCH1501 Investigation Workshop (UNSW, FBE, C. Lassen and M. Gusheh course conveners) is taken by final year Architecture students and is the precursor course to their Graduation Project. At the beginning of the session the entire cohort of students is presented with a range of studio group options that reflect the particular research interests of the staff involved and they enter a ballot as to their preferred option. In the studio group option this author presented students would be introduced to workshops held in conjunction with the tenth artificial LIFE conference held in June 2006 and asked to use what they understood to be the intention of one workshop to engage with a series of architectural issues.

In Picon and Ponte's introduction to Architecture and the Sciences: Exchanging Metaphors they refer to a new type of enterprise "in which the sciences are viewed not from the interior, as is usual, but from the exterior - that is, from sites and places interfacing with other fields of knowledge and culture."

The attitude to biotechnology developed in this course borrows from the idea of viewing sciences from the exterior but rejects the implication in the books subtitle that viewing a subject from the site of another necessitates a purely metaphorical understanding. The United Nations Convention on Biological Diversity defines biotechnology as "any technological application that uses biological systems, living organisms, or derivatives thereof, to make or modify products or processes for specific use." The latest computer gaming technology (used in this studio) with its real time simulation of many of the physical parameters shared between the architecture of the built world and the architecture of the biological world gave students the opportunity to experiment in the intersection between them in a very direct way, i.e. with a unprecedented amount of realism, interactivity and testability (this incredible new capability in computer games has only been available since 2005, with Valve's HL2). Using computer gaming technology that has integrated real world physics to engage with some of the ideas within biotechnology extends Frost's (2002) understanding of the design sketch as a "boundary object". Frost understands Leigh Star's (Star 1989) concept of the boundary object in science as a material object that facilitates the coordination of scientific work because they can be simultaneously read by generalists and specialists. Even though the focus of their mutual attention may be quite different the boundary object allows them to come together for some common endeavour. The environments of these new computer games, enabled with real world physics, allowed the "generalist" architectural design students in this course to directly negotiate with ideas within biotechnology from an understanding of architectural space they related to implicitly. It is this capability that distinguishes the design studio task here from Picon and Ponte's exchanging of metaphors. 


\section{ARCH1501IW aLIFEx student work}

To give a sense of a progression through that series I will describe 3 experiments by one student, Shawn Li, with additional comments as captions to the work of Andrew Lim, Andrew Wallace, Cheung Lok Kan and George Barbas. In addition to the submission of their real time environments the students were required to represent there findings in short video animations. The comments below directly reference those animations. Please find a link to the animations below (Lowe, 2007).

1. Shawn Li, EXP1: The Workshop. In the representation of this experiment Shawn develops a narrative that introduces a character grappling with the concept of the 'boundary object'. His character explores the instruments available in the HL2 modification GMod to alter visual and material reality. "Bit by bit he would piece together a contraption that would grant him passage out of the void". Over the period of the animation Shawn's workshop transforms from a place of passive experience to a place of actual fabrication, i.e. the materiality of objects, new relationships between objects, and between the observer and environment, are created and altered within the real time environment.

2. Shawn Li, EXP2: The Differential. The premise of this experiment suggested that within extremely complex sequences control becomes an act of shepherding rather than ridged prescription (Lowe 2007). The Architectural challenge was to create a physical system that I called a "Differential". The Differential received inputs and through a sequence of operations produced an output. "The key to differentials is the relationship between two entities with a common stimulus. This relationship is a compensatory one that often includes an action and reaction. The role of this in the artificial life is the understanding that its methods will bring about different effects on its surrounding (during the process) even if the end result is similar." Shawn Li.

3. Shawn Li, EXP3: The Physical Collaboration. In the final experiment Shawn's hypothesis was that the architecture of a path through a space could influence the user of that pathways contribution to group collaboration. The collaborative activity involved the manipulation of a 5 story tall marionette so that it would 'walk' down the length of the space. Each participant physically controlled a key joint in the marionettes structure and followed a path that sloped up and down with the intention of promoting their intended contribution. As you can see in the video capture of the actual event the overall control of the marionette becomes less as the number of controlling participants is increased. While Shawn's strategy seemed reasonable at the outset, and was reasonable within a limited range (1-2 participants), the real time collaborative experienced suggested that the architecture would have to be much more sophisticated than it was to make the contribution ultimately intended. That the architecture needed to promote/accommodate more sophisticated communications networks, and possibly a central brain, was another outcome of the experiment.

Andrew Lim: Architecture as avatar, the development of a non anthropomorphic participant.

Andrew Wallace: A rhythm of linear and rotational motion is destabilised as zones of tolerance become so broad as to overlap.

George Barbas: An environment that evolves through responding to the presence and movement of its inhabitants.

Cheung Lok Kan: "The physical impossibility of death in the (artificial) mind of someone living". Damien Hirst (1991).

\section{Conclusion}

The last few years (since 2003) have seen a major shift in the approach computer game designers have taken with respect to the integration of custom content in their environment. Along with outsourcing for geometry and materiality, to practitioners of CAAD, they are actively moving away from the need to program within their world building interfaces. Both of these trends are an advantage for students and practitioners of Architecture. Taking advantage of the strength of solid modelling software, not traditionally used by architects, to remove a large source of errors in the integration process streamlined that process significantly with respect to previous authors experience. Combine these factors and even relatively inexperienced students of architecture are afforded much greater ability to design and test their spaces, materiality and interactivity in real time (with all of the benefits Frost, Hoon, Kehoe, Lehtinen, Moloney etc have 
previously described). In contrast to previous applications of computer gaming technology within architectural curriculum's the introduction of real time physics engines results in architectures responding in a much more sophisticated way to the interaction and experience of their occupants. Affording a direct engagement with disciplines outside of architecture, through computer gaming technology as 'boundary object', introduces an important quantifiable supplement to the traditional qualitative exchange of metaphors.

\section{References}

3dBuzz. [http://www.3dbuzz.com/vbforum/sv home.php]

Achten, $\mathrm{H}$ and Turksma A. Virtual Reality in Early Design: The Design Studio Experience. 1999

aLIFEx. http://www.alifex.org/ . [http://www.alifex.org/workshops/]

Eigen, Edward. Architecture and the Sciences. Ed. Antoine Picon and Alessandra Ponte. Princeton Architectural Press. 2003. 62.

Frascati. "Main definitions and conventions for the measurement of research and experimental development (r\&d)." A summary of the Frascati manual 1993. Organization for economic cooperation and development. Paris 1994. 7

[http://www1.oecd.org/dsti/sti/stat-ana/prod/eas fras.htm]

GMod. A Modification of Valve's HL2 [http://garrysmod.com/]

Hoon, M and Kehoe, M. Enhancing Architectural Communication with Gaming Engines. Acadia 22 >> Connecting Crossroads of Digital Discourse. 350

“Imagina: games lead R\&D”. 3D World. April 2007. 20

Krause, Rosalind E. The Originality of the Avant-Garde and Other Modernist Myths. Cambridge. Mass. MIT Press. 1985.

Lehtinen, S. Visualization and teaching with state-of-the-art 3D game technologies. eCAADe 20 [design e-ducation] Modeling Real and Virtual Worlds Session 14. 538-541

Lowe, R. "DESN104: Introduction to Computers for Designers." Nov. 2006. [http://www.russelllowe.com/desn104 2006t2/index.htm].

Lowe, R. “ARCH1501 IW aLIFEx. June 2007.

[ http://www.russelllowe.com/arch1501/student work 2007.htm ]

Matta-Clark, Gordon. Interview with Donald Wall. "Gordon Matta-Clark's Building Dissections". Arts Magazine. May 1976. Arts Digest. 74-79.

Moloney, J. String CVE, Collaborative Virtual Environment software developed from a game engine. eCAADe 20 [design e-ducation] Modeling Real and Virtual Worlds Session 14. 5225252002

Picon and Ponte, eds, Architecture and the Sciences: Exchanging Metaphors, Princeton Papers on Architecture, Princeton Architectural Press, 2003. 13

Stratton, John. "Surveying Synthetic Instruments for Defence." February 2005. http://www.mwrf.com/Articles/ArticleID/9795/9795.html recovered 18 April 2007

"Student Work DESN104" [http://www.russelllowe.com/desn1042006t2/experiment3/reference/student work.html]

"Student Work ARCH1501 Investigation Workshop". [www.russelllowe.com/arch1501/student work.html]

Treadwell, S. In conversation with the author, Auckland University School of Architecture, NZ 1996.

United Nations. "The Convention on Biological Diversity (Article 2. Use of Terms)."1992. Retrieved on September 20, 2006.

UNSW FBE (University of New South Wales, Faculty of the Built Environment).

"Urban Dictionary" [http://www.urbandictionary.com]

"Unreal Technology" http://www.unrealtechnology.com/html/technology/ue30.shtml recovered 25 April 2007 
"Valve". Computer Game Developer, Half Life 2 (HL2)

[http://developer.valvesoftware.com/wiki/Category:Hammer] 